\title{
Contamination des eaux souterraines par le lixiviat des décharges publiques : Cas de la nappe phréatique R'Mel (Province de Larache - Maroc Nord-Occidental)
}

\author{
H. ER-RAIOUI ${ }^{1 *}$, S. BOUZID ${ }^{1}$, S. KHANNOUS ${ }^{1}$ et M. A. ZOUAG ${ }^{1}$ \\ ${ }^{I}$ Equipe " Géosciences et Environnement »- Faculté des Sciences et Techniques à Tanger - Université \\ Abdelmalek Essâadi- Ancienne route de l'aviation, BP. 416, Tanger, Maroc. Tél : (00212) 393939 54, \\ Fax : (00212) 0393939 53, e-mail : h.erraioui@fstt.ac.ma. \\ *Auteur correspondant, E-mail : h.erraioui@fstt.ac.ma
}

\section{RESUME}

La percolation du lixiviat en provenance des déchets des décharges constitue une source de pollution des ressources en eau souterraine par infiltration, constituant ainsi une menace aussi importante que celle liée à l'accentuation du déficit hydrique. La décharge publique de Larache constitue un modèle type de détérioration des ressources en eaux par un effet de contamination du lixiviat en provenance de la décharge et des pratiques agricoles. Les mesures réalisées sur le lixiviat et sur les eaux prélevées des puits situés autour de la décharge et ayant porté sur les paramètres physicochimiques, de pollution $\left(\mathrm{O}_{2}\right.$ dissous, DCO...), de métaux lourds et sur des analyses bactériologiques, permettent de qualifier le lixiviat comme une source de contamination à potentiel anoxique et réducteur. La charge polluante est importante et se traduit par des valeurs de DCO et d'azote kjeldahl élevées $(63375 \mathrm{mg} / \mathrm{l}$ et de $798 \mathrm{mg} / \mathrm{l})$. Les résultats permettent aussi de conclure sur la qualité médiocre des eaux contenues dans les poches souterraines notamment pendant la période sèche, ce qui est illustrée par les valeurs élevées en certains métaux ( $\mathrm{Pb}(800 \mu \mathrm{g} / \mathrm{l}), \mathrm{Cd}(40 \mu \mathrm{g} / \mathrm{l}), \mathrm{Cr}(80 \mu \mathrm{g} / \mathrm{l})$ et $\mathrm{Fe}(650 \mathrm{mg} / \mathrm{l}))$, en coliformes totaux, en coliformes fécaux et en streptocoques fécaux.

(c) 2011 International Formulae Group. All rights reserved.

Mots clés : Charge polluante, paramètres physico-chimiques, sels nutritifs, métaux, bactériologie, lixiviat.

\section{INTRODUCTION}

Inhérentes au développement socioéconomique, qui ne peut se faire sans effets néfastes sur l'environnement et l'économie de nombreux pays (Baun et al., 2000; Christensen et al., 2001 ; Khattabi, 2002), les sources potentielles de pollution des eaux souterraines se sont multipliées au cours des dernières années (Danielpol et al., 2003). Parmi les contaminants les plus répandus, les nitrates occupent une place importante puisqu'ils sont produits par les activités humaines de base (agriculture, activités domestiques, industries, etc.). Une concentration élevée du nitrate en eau potable peut être pathologique pour des humains et des animaux (Kross et al., 1992). Au Maroc, la contamination des eaux souterraines a suscité, ces dernières années, une attention particulière en raison des problèmes causés par la dégradation de plus en plus importante de la qualité de l'eau des nappes par les nitrates essentiellement (Hudak, 2000 ; Harter et al., 2002; Laftouhi et al., 2003). 
L'évolution démographique et l'intensification des activités économiques sont accompagnées d'une augmentation de la production des déchets, estimés à 4,5 millions de tonnes / an de déchets urbains et 800000 tonnes /an des déchets industriels. Cela conduit à une moyenne de $1 \mathrm{~kg}$ de déchets par jour et par habitant (Naifar, 1996 ; Amhoud, 1997). Ces déchets sont variés et admettent ce qui est industriel, hospitalier entre autres et dont les nuisances deviennent inquiétantes.

La percolation des eaux à travers ces déchets provoque la production des lixiviats qui se chargent en matières organiques, bactéries, composés minéraux et en métaux lourds pour l'essentiel. Les lixiviats qui constituent le principal vecteur de transport de la pollution métallique issue des sites de stockage des déchets (Jourdan et al., 2005), présentent une composition très aléatoire et varie en fonction de la nature des déchets, l'âge de la décharge, la technique d'exploitation et les conditions climatiques. La fraction minérale des lixiviats de décharge est constituée essentiellement de chlorures, sulfates, bicarbonates, potassium, sodium et ammonium, alors que la partie organique est très liée à l'âge des déchets. Si les jeunes décharges sont caractérisées par la dominance des acides gras volatiles (AGV) (surtout les acides acétiques, propioniques et butyriques), ce sont des substances assimilées aux composés fulviques et humiques qui sont prépondérantes dans les lixiviats âgés (Christensen et al., 2001). Le volume du lixiviat et son infiltration vers les eaux du fond reste tributaire des précipitations et de la perméabilité du plancher couvrant les nappes phréatiques.

Dans le secteur d'étude, l'analyse géologique, géographique et hydroclimatologique permet de mettre le point sur l'importance de la perméabilité des formations constituant le plateau de la nappe R'Mel. Ceci favorise l'infiltration des eaux de pluie dans le secteur caractérisé par une pluviométrie annuelle avoisinant $700 \mathrm{~mm}$ (ABHL, 2003) constituant ainsi, un paramètre en faveur de la production de quantités importantes de lixiviat et par la suite la contamination des eaux souterraines. Les coefficients de ruissellement et d'infiltration sont respectivement de l'ordre de $10 \%$ et de $37 \%$. La carte de la profondeur de la nappe par rapport au sol, établie à partir des données de 39 piézomètres relevés lors d'une campagne de mesure de l'agence du bassin hydraulique Loukkos (ABHL, 2003), montre un niveau d'eau qui varie, dans l'ensemble, entre 5 et $20 \mathrm{~m}$. Les eaux sont utilisées pour la production des eaux potables, l'abreuvage des animaux et surtout pour l'irrigation des cultures dans la région, ce qui rend la situation plus compliquée. A l'effet du lixiviat peut s'ajouter l'influence des pratiques agricoles et des eaux des égouts domestiques, directement déchargées dans la nature sans traitement préalable suite à l'absence ou au disfonctionnement des stations d'épuration.

Située au Nord-Ouest du Maroc (Figure 1), la ville de Larache a une population de 116 971 habitants. La production journalière des déchets est de $81 \mathrm{t} / \mathrm{j}$, ce qui implique une production annuelle de 29565 t/an. Les principales activités économiques de la zone sont l'agriculture et l'industrie agroalimentaire. Les principales cultures pratiquées dans cette région sont les agrumes, la canne à sucre et les cultures maraîchères. Les déchets sont constitués de déchets ménagers, déchets industriels (conserveries de légumes, conserveries de poissons, produits périmés de l'industrie agroalimentaire, déchets métalliques provenant de l'usine de pièces, déchets de construction, déchets de dégraissage et de vidange, déchets d'abattoirs, déchets d'industrie de cuir, déchets de textile) et hospitaliers. La décharge publique, de type sauvage, a été mise en fonction au début des années 1988. Le calcul avec le logiciel Autocad montre une superficie de 4,71 ha. La mauvaise gestion et l'absence d'un contrôle efficace constituent une véritable menace de contamination pour les trois composantes de l'environnement contaminant (air, sol et ressources en eau).

$$
\text { Pour une caractérisation }
$$

physicochimique et biogéochimique du lixiviat et son impact sur la nappe phréatique 
R'Mel qui paraît évident, la présente étude étaye sur la réalisation d'analyses portant sur les lixiviats libérés par les ordures et sur les eaux des puits qui se situent autour de la décharge en vue de mettre l'accent sur le degré de contamination des eaux à proximité de la décharge.

\section{MATERIEL ET METHODES}

L'échantillonnage intéressant les eaux de puits et le lixiviat a été mené en deux périodes distinctes. La première période a eu lieu après les fortes pluies du printemps 2006 (période humide) et la deuxième a été réalisée pendant la période juillet-Août 2006, lors d'une période sèche. Le lixiviat a été prélevé au niveau des écoulements près des déchets. Parallèlement, six prélèvements ont été opérés dans des puits situés autour de la décharge afin d'analyser l'état de dégradation de la nappe phréatique avoisinante la décharge publique (Figure 2). En ce qui concerne la localisation, à l'exception du puits P6 situé en plein air, les autres sources d'eau, au nombre de 5, sont localisées au sein des fermes. Les échantillons d'eau ont été prélevés dans des pots en verre pré-nettoyés selon le processus de Rodier (1996) et placés à $4{ }^{\circ} \mathrm{C}$ dans une glacière. Les analyses ont été effectuées dès le retour au laboratoire. Les données de température, de $\mathrm{pH}$, d'oxygène dissous, de conductivité ont été mesurées in situ au moment du prélèvement de l'échantillon à l'aide d'un multi paramètre portatif. La détermination des matières en suspension (MES) a été effectuée par filtration d'un volume connu d'eau. La demande chimique en oxygène (DCO) est déterminée par une oxydation au bichromate de potassium en milieu acide et en présence de sulfate d'argent et de mercure (Rodier, 1996). Les phosphates et les nitrates ont été déterminés par spectrophotométrie. Les Sulfates, les chlorures, le calcium et le magnésium ont été analysés par méthode gravimétrique. Les éléments Sodium et Potassium par Photométrie de flamme et les éléments Plomb, Cuivre, Zinc, Chrome VI, Cadmium et Cobalt par Adsorption atomique. Les coliformes totaux et fécaux ainsi que les streptocoques fécaux ont été déterminés par dénombrement. Toutes les manipulations bactériologiques ont été effectuées dans une zone stérilisée. Une prise d'essai de $1 \mathrm{ml}$ d'eau est ajoutée à $9 \mathrm{ml}$ de diluant stérile (Tryptone-sel). L'analyse statistique adoptée correspond au programme SPSS qui permet de mettre l'accent sur les liens et les variations pouvant exister entre les paramètres étudiés. Les corrélations ont été établies grâce au coéficient de corrélation des variables Spearman. L'analyse des variations des différents paramètres a été réalisée par l'analyse de variance (ANOVA) qui permet d'évaluer la variance à travers le paramètre $\mathrm{F}$ de Fissher (rapport carrés moyens groupes/carrés moyens erreurs) et le degré de signification $\mathrm{p}$ (probabilité d'être hors intervalle de confiance).

\section{RESULTATS}

Les échantillons de lixiviat et d'eau prélevés des puits situés autour de la décharge ont fait l'objet d'analyses physique, biogéochimique et bactériologique. Les résultats dégagés et correspondant aux valeurs moyennes sont consignés dans les Tableaux 1, $2,3,4$ et 5 .

\section{Analyse du lixiviat}

Les données relatives au $\mathrm{pH}$ montrent que le lixiviat présente des caractéristiques différentes selon les saisons (Tableaux 1 et 2). Pendant la période humide, le lixiviat est légèrement basique avec un $\mathrm{pH}$ de 7,87 alors que pour la période sèche, le lixiviat est acide et montre un $\mathrm{pH}$ de 4,78. Les concentrations relatives aux taux d'oxygène dissous sont négligeables. Les valeurs sont inférieures à $0,2 \mathrm{mg} / \mathrm{l}$, ce qui permet de qualifier le lixiviat comme un milieu anoxique presque dépourvu d'oxygène libre et tend à être réducteur vu l'importance de la charge organique oxydable. Les résultats dégagés correspondant à la demande chimique en oxygène (DCO) montrent des valeurs qui varient de 44750 $\mathrm{mg} / \mathrm{l}$ lors de la période sèche à $82000 \mathrm{mg} / \mathrm{l}$ lors de la période humide (Tableaux 1 et 2). Ces concentrations élevées, évoquent la 
présence de quantités importantes de matières oxydables constituant ainsi une importante demande en oxygène pour son oxydation, mettant en jeu l'offre du milieu. Pour la conductivité, les valeurs relevées sont assez élevées. Les résultats montrent une valeur de $64.6 \mathrm{~ms} / \mathrm{cm}$ pendant la période humide et de $148.75 \mathrm{~ms} / \mathrm{cm}$ lors de la période sèche, ce qui peut être expliqué par le bilan ionique du lixiviat. En effet, les concentrations en sulfates, $\mathrm{Ca}^{2+}, \mathrm{Mg}^{2+}, \mathrm{Cl}^{-}, \mathrm{K}^{+}$et $\mathrm{Na}^{+}$sont très élevées et varient de la période humide à la période sèche. Les degrés de signification des variations sont typiques $(p \leq 0,05)$. Les valeurs fluctuent de $240 \mathrm{mg} / \mathrm{l}$ à $336 \mathrm{mg} / \mathrm{l}$ pour les sulfates, de $450 \mathrm{mg} / \mathrm{l}$ à $6000 \mathrm{mg} / \mathrm{l}$ pour le calcium, de $240 \mathrm{mg} / \mathrm{l}$ à $2100 \mathrm{mg} / \mathrm{l}$ pour le magnésium, de $18415 \mathrm{mg} / \mathrm{l}$ à $70955 \mathrm{mg} / \mathrm{l}$ pour les chlorures, de $2613 \mathrm{mg} / \mathrm{l}$ à $14365 \mathrm{mg} / \mathrm{l}$ pour le potassium et de $14260 \mathrm{mg} / \mathrm{l}$ à $27968 \mathrm{mg} / \mathrm{l}$ pour le sodium. Les concentrations relatives à la période sèche sont plus élevées que celles relevées lors de la période humide. L'azote kjeldahl montre des concentrations presque constantes entre la période humide et la période sèche avec des valeurs respectives de $795.2 \mathrm{mg} / \mathrm{l}$ et $800.2 \mathrm{mg} / \mathrm{l}$ (Tableaux 1 et 2). Le fer, le $\mathrm{Pb}$ et le $\mathrm{Cd}$ montrent des concentrations respectives de $16650 \mu \mathrm{g} / \mathrm{l}, 280 \mu \mathrm{g} / \mathrm{l}$ et $30 \mu \mathrm{g} / \mathrm{l}$. Pour la bactériologie, seuls les échantillons de la période sèche ont fait l'objet d'analyses. Les résultats sont de l'ordre de 316000 UFC/100 ml pour les coliformes totaux, $152000 \mathrm{UFC} / 100 \mathrm{ml}$ pour les coliformes fécaux et $875 \mathrm{UFC} / 100 \mathrm{ml}$ pour les streptocoques fécaux (Tableau 5). Le nombre de bactéries est élevé et reflète un milieu contaminé. Les autres paramètres déterminés et relatives aux MES, phosphates, nitrates, cuivre, cadmium, cobalt, zinc et chrome ne présentent pas de valeurs significatives mettant en cause la qualité des eaux du lixiviat.

\section{Analyse des eaux de puits}

Les analyses effectuées au niveau des puits montrent des eaux bien oxygénées. Les teneurs en oxygène dissous varient de 6 à 9,3 $\mathrm{mg} / \mathrm{l}$. Les eaux sont aussi peu chargées en matières oxydables, ce qui explique l'oxygénation des eaux des puits. Les valeurs de DCO sont souvent inférieures à la limite admise $(25 \mathrm{mg} / \mathrm{l})$ à l'exception des eaux des puits P3 et P6 qui affichent des DCO de 32 $\mathrm{mg} / \mathrm{l}$ et de $44 \mathrm{mg} / \mathrm{l}$ respectivement. La conductivité électrique varie de 0,73 à 1,15 $\mathrm{ms} / \mathrm{cm}$. Les résultats correspondant aux nitrates montrent des valeurs qui fluctuent entre 52 et $97 \mathrm{mg} / \mathrm{l}$ pour la période humide et entre $46 \mathrm{mg} / \mathrm{l}$ et $114 \mathrm{mg} / \mathrm{l}$ pour la période sèche. Les maximums sont enregistrés au niveau des puits P3, P4, P5 et P6 avec des valeurs qui dépassent $80 \mathrm{mg} / \mathrm{l}$ pour les deux périodes. La variation des concentrations dans le temps est peu significative $(\mathrm{P}=0,98)$. Les chlorures montrent des variations assez significatives entre la période humide et sèche $(\mathrm{p}=0,05)$. Les concentrations relevées lors de la période humide présentent un maximum de $159 \mathrm{mg} / \mathrm{l}$ alors que celles de la période sèche dépassent, par endroit, $200 \mathrm{mg} / \mathrm{l}$. Les concentrations en sulfates varient peu entre les deux saisons et montrent des valeurs inférieures à $250 \mathrm{mg} / \mathrm{l}$. Les phosphates sont en traces au niveau de tous les puits. Pour le calcium et pendant la période humide, les puits P4 et P6 présentent des concentrations respectives de $162 \mathrm{mg} / \mathrm{l}$ et $184 \mathrm{mg} / \mathrm{l}$. Pour la période sèche, les concentrations en calcium sont élevées au niveau de tous les puits et fluctuent de $192 \mathrm{mg} / \mathrm{l}$ à $252 \mathrm{mg} / \mathrm{l}$ (Tableaux 3 et 4). Pour la variation des concentrations dans le temps, les valeurs relevées lors de la période sèche sont plus élevées que celles enregistrées lors de la période humide. Pour les éléments $\mathrm{K}+, \mathrm{Na}+$ et $\mathrm{Mg}++$, les concentrations déterminées au niveau de tous les puits restent inférieures aux normes admises. Exprimées en $\mathrm{mg} / \mathrm{l}$, les concentrations sont respectivement de 2,97, 58,88 et 10,80 .

Les résultats relatifs aux métaux analysés ( $\mathrm{Pb}, \mathrm{Cd}, \mathrm{Cr}, \mathrm{Zn}, \mathrm{Cu}, \mathrm{Co}$ et $\mathrm{Fe})$ montrent des concentrations en $\mathrm{Pb}$ et $\mathrm{Cd}$ qui varient respectivement de 230 à $800 \mu \mathrm{g} / \mathrm{l}$ et de 10 à $40 \mu \mathrm{g} / \mathrm{l}$. Pour les éléments $\mathrm{Cr}$ et $\mathrm{Fe}$, les concentrations les plus élevées sont de 70 et $80 \mu \mathrm{g} / \mathrm{l}$ pour le $\mathrm{Cr}$, enregistrées au niveau des puits P3 et P6, et de 650 et $300 \mu \mathrm{g} / \mathrm{l}$ pour le 
fer, enregistrées au niveau des puits $\mathrm{P} 2$ et $\mathrm{P} 5$ (Tableau 3). Les autres éléments $\mathrm{Co}, \mathrm{Zn}$ et $\mathrm{Cu}$ montrent des teneurs respectives de $40 \mu \mathrm{g} / \mathrm{l}$, $10 \mu \mathrm{g} / \mathrm{l}$ et $340 \mu \mathrm{g} / \mathrm{l}$. Le dénombrement des bactéries montrent des valeurs qui fluctuent entre $2050 \mathrm{UFC} / 100 \mathrm{ml}$ et $33400 \mathrm{UFC} / 100 \mathrm{ml}$ pour les coliformes totaux. Le maximum de $33400 \mathrm{UFC} / 100 \mathrm{ml}$ est enregistré au niveau du puits P6. Pour les coliformes fécaux, les valeurs varient de $500 \mathrm{UFC} / 100 \mathrm{ml}$ à 13966 UFC/100 ml, enregistrées au niveau du puits P5 et pour les streptocoques fécaux, les valeurs varient entre $100 \mathrm{UFC} / 100 \mathrm{ml}$ et $68000 \mathrm{UFC} / 100 \mathrm{ml}$, enregistrées au niveau du puits P1 (Tableau 5).
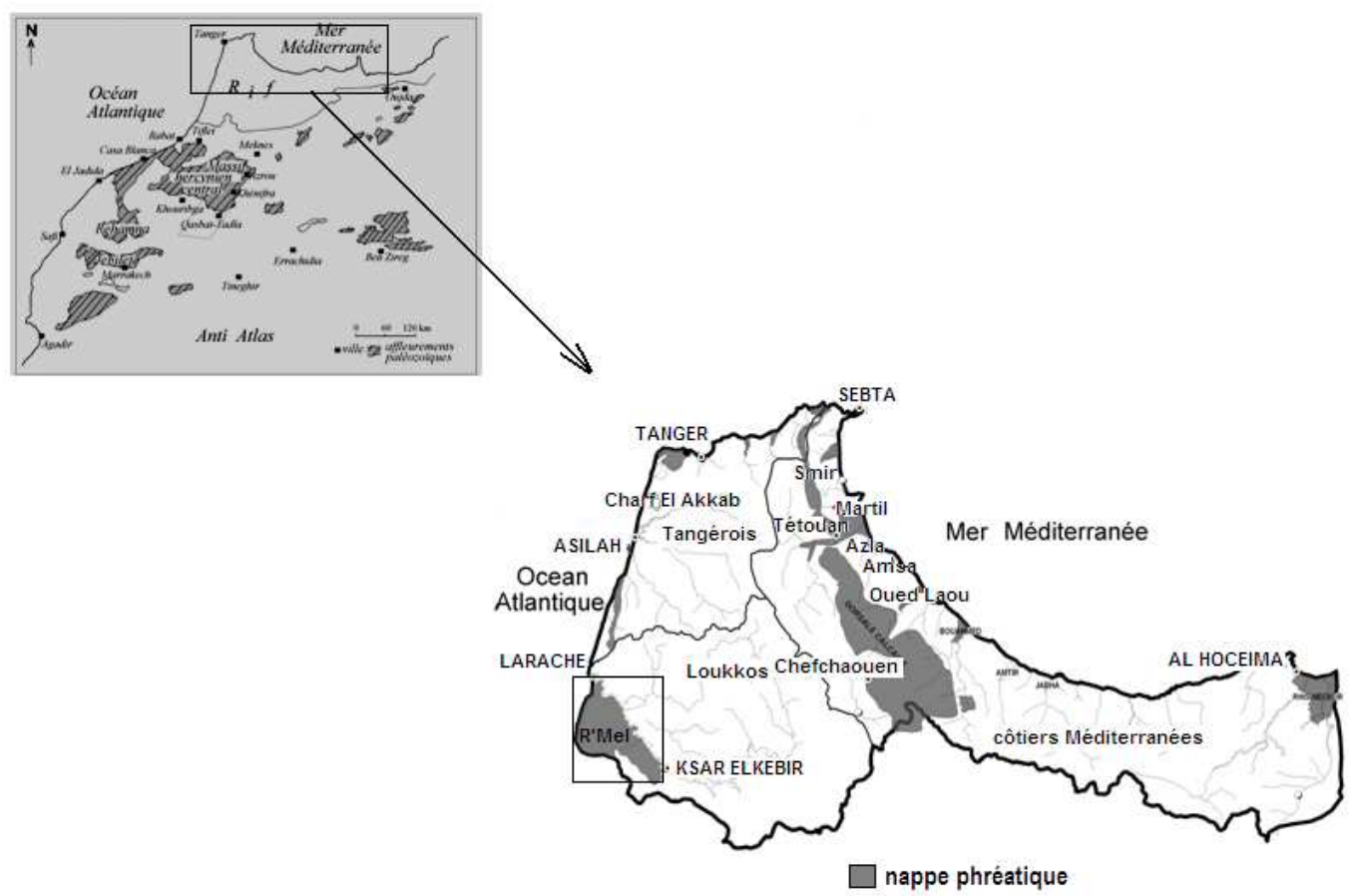

Figure 1 : Situation de la zone d'étude (nappe phréatique de Larache)

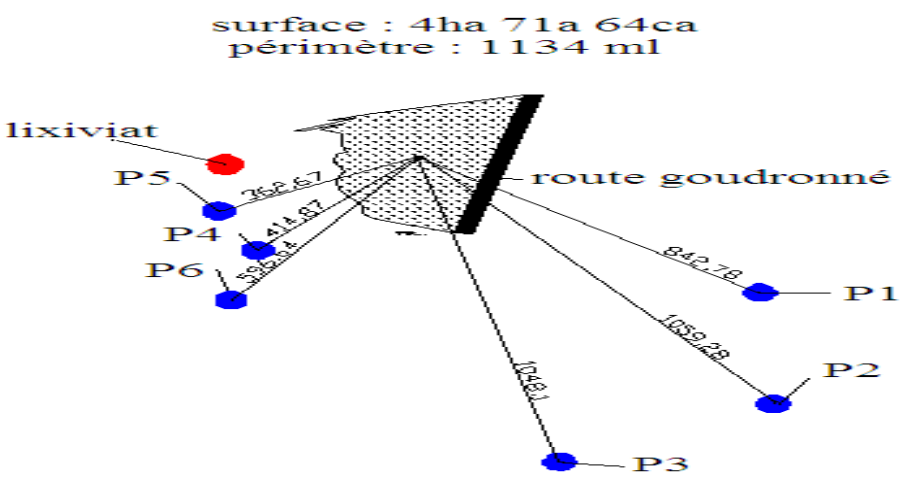

Figure 2 : Positionnement des puits analysés par rapport à la décharge publique de la ville de Larache. 


\section{H. ER-RAIOUI et al. / Int. J. Biol. Chem. Sci. 5(3): 1118-1134, 2011}

Tableau 1 : résultats physico-chimiques et bio-géochimiques du lixiviat et des eaux des puits (période humide) (exprimées en mg/l) et normes marocaines.

\begin{tabular}{|c|c|c|c|c|c|c|c|c|c|c|c|}
\hline Site & $\mathbf{T}^{\circ} \mathbf{C}$ & pH & MES & Od & $\begin{array}{l}\text { Cond } \\
\mathrm{ms} / \mathrm{cm}\end{array}$ & DCO & Nitrates & $\begin{array}{c}\text { Azote } \\
\text { Kjeldhal }\end{array}$ & Chlorures & Sulfates & Phosphates \\
\hline Lixiviat & 24 & 7.87 & 4.07 & 0.2 & 64.6 & $44750 \pm 166,43$ & - & 795 & $\begin{array}{l}18415 \pm \\
758,07\end{array}$ & 240 & 0.56 \\
\hline $\mathbf{P 1}$ & 23.20 & 6.96 & 0.25 & 7.6 & 0.83 & $12 \pm 2,65$ & $62 \pm 8,08$ & 2.80 & 159.80 & 14.40 & traces \\
\hline $\mathbf{P 2}$ & 19.90 & 6.91 & 0.17 & 8.6 & 0.83 & $8 \pm 1$ & $52 \pm 2,65$ & 4.20 & 150.90 & 19.20 & traces \\
\hline $\mathbf{P 3}$ & 20.30 & 7.15 & 0.24 & 7.4 & 0.71 & $32 \pm 5,20$ & $83 \pm 9,85$ & 3.50 & 97.60 & 28.80 & traces \\
\hline $\mathbf{P 4}$ & 19.30 & 7.26 & 0.28 & 6.3 & 1.01 & $12 \pm 2,65$ & $91 \pm 5,00$ & 4.20 & 124.30 & 67.20 & traces \\
\hline P5 & 19.60 & 7.05 & 0.24 & 6.9 & 0.93 & $10 \pm 3$ & $97 \pm 6,24$ & 5.60 & 106.50 & 28.80 & traces \\
\hline P6 & 21.60 & 6.68 & 0.19 & 9.3 & 1.15 & $44 \pm 2,52$ & $97 \pm 7,00$ & 4.20 & 159.80 & 52.80 & traces \\
\hline Normes & - & - & - & - & 2,5 & 25 & 50 & - & 200 & 250 & 0,7 \\
\hline
\end{tabular}

$\mathrm{T}^{\circ}$ : température, $\mathrm{pH}$ : potentiel d'hydrogène, MES : matière en suspension, Od : oxygène dissous, Cond : conductivité, DCO : demande chimique en oxygène.

Tableau 2 : résultats physico-chimiques et bio-géochimiques du lixiviat et des eaux des puits (période sèche) (exprimées en mg/l).

\begin{tabular}{ccccccccccccc}
\hline Site & $\mathbf{T}^{\circ} \mathbf{C}$ & $\mathbf{p H}$ & MES & $\mathbf{O d} \begin{array}{c}\text { Cond } \\
\mathbf{m s} / \mathbf{c m}\end{array}$ & DCO & Nitrates & $\begin{array}{c}\text { Azote } \\
\text { Kjeldhal }\end{array}$ & Chlorures & Sulfates Phosphates \\
\hline Lixiviat & 33 & 4.78 & 5.12 & 0.1 & 149 & $82000 \pm 470,88$ & $23 \pm 3,61$ & 800 & & 336 & 0.61 \\
P1 & 23.70 & 6.58 & 0.27 & 6.40 & 0.87 & $24 \pm 7,21$ & $59 \pm 6,08$ & 3.50 & $221.88 \pm 17,64$ & 26.40 & traces \\
& & & & & & & & & & & &
\end{tabular}


H. ER-RAIOUI et al. / Int. J. Biol. Chem. Sci. 5(3): 1118-1134, 2011

\begin{tabular}{ccccccccccccc}
\hline $\mathbf{P 2}$ & 20.20 & 6.59 & 0.14 & 6.90 & 0.82 & $18 \pm 2$ & $46 \pm 6,08$ & 5.60 & $239.63 \pm 19,45$ & 28.80 & traces \\
P3 & 21 & 6.66 & 0.32 & 6.40 & 0.73 & $20 \pm 4$ & $84 \pm 3,61$ & 3.50 & $177.50 \pm 9,01$ & 40.80 & traces \\
$\mathbf{P 4}$ & 23.30 & 6.86 & 0.20 & 6.10 & 0.97 & $20 \pm 3,46$ & $88 \pm 5,29$ & 2.80 & $159.75 \pm 7,78$ & 74.40 & traces \\
P5 & 21.70 & 6.69 & 0.27 & 6 & 0.97 & $16 \pm 3,46$ & $102 \pm 9,17$ & 4.20 & $195.25 \pm 30,85$ & 36 & traces \\
P6 & 25 & 7.04 & 0.21 & 8.20 & 1.12 & $20 \pm 4,36$ & $114 \pm 11,72$ & 5.60 & $248.50 \pm 7,70$ & 55.20 & traces
\end{tabular}

$\mathrm{T}^{\circ}$ : température, $\mathrm{pH}$ : potentiel d'hydrogène, MES : matière en suspension, Od : oxygène dissous, Cond : conductivité, DCO : demande chimique en oxygène.

Tableau 3 : résultats des analyses des ions métalliques des eaux des puits (période humide) (exprimées en mg/l) et normes marocaines

\begin{tabular}{cccccccccccc}
\hline Site & $\mathbf{C a}^{+}$ & $\mathbf{K}^{+}$ & $\mathbf{N a}^{+}$ & $\mathbf{F e}$ & $\mathbf{C u}$ & $\mathbf{Z n}$ & $\mathbf{P b}$ & $\mathbf{C d}$ & $\mathbf{C r} \mathbf{6}$ & $\mathbf{M g}^{2+}$ & $\mathbf{C o}$ \\
\hline Lixiviat & 450 & 2613 & 14260 & $16.65 \pm 4$ & 0.15 & 0.34 & $0.28 \pm 0,08$ & $0.03 \pm 0,01$ & 0.03 & 240 & 0.04 \\
P1 & 127 & 2.50 & 48.76 & $0.05 \pm 0,02$ & - & 0.08 & $0.23 \pm 0,05$ & $0.01 \pm 0,01$ & 0.02 & 6.72 & 0.01 \\
P2 & 135 & 1.56 & 37.72 & $0.65 \pm 0,10$ & 0.03 & 0.05 & $0.57 \pm 0,06$ & $0.02 \pm 0,01$ & 0.04 & 7.92 & - \\
P3 & 116 & 1.01 & 28.06 & $0.14 \pm 0,02$ & 0.01 & 0.04 & $0.42 \pm 0,03$ & $0.01 \pm 0,01$ & 0.07 & 7.92 & - \\
P4 & 162 & 1.72 & 50.60 & $0.01 \pm 0,01$ & 0.02 & 0.03 & $0.29 \pm 0,13$ & $0.01 \pm 0,01$ & 0.04 & 10.80 & - \\
P5 & 153 & 1.01 & 43.24 & $0.30 \pm 0,09$ & 0.34 & 0.01 & $0.28 \pm 0,09$ & $0.03 \pm 0,01$ & 0.01 & 6.72 & 0.04 \\
P6 & 184 & 1.56 & 58.88 & $0.08 \pm 0,01$ & 0.02 & 0.02 & $0.79 \pm 0,04$ & $0.04 \pm 0,02$ & 0.08 & 4.80 & - \\
Normes & 160 & 12 & 200 & 0,2 & 0,5 & 5 & 0,01 & 0,005 & 0,05 & 50 & \\
\hline
\end{tabular}


H. ER-RAIOUI et al. / Int. J. Biol. Chem. Sci. 5(3): 1118-1134, 2011

Tableau 4 : résultats des analyses des ions métalliques des eaux des puits (période sèche) (exprimées en mg/l).

\begin{tabular}{cccccccc}
\hline & Lixiviat & P1 & P2 & P3 & P4 & P5 & P6 \\
\hline Ca+ & 6000 & 192 & 194 & 170 & 220 & 222 & 252 \\
K+ & 14365 & 2,97 & 1,40 & 0,94 & 1,56 & 1,56 & 2,34 \\
Na+ & 7968 & 51,89 & 37,54 & 31,10 & 48,21 & 45,26 & 55,94 \\
Mg++ & 2100 & 9,6 & 1,2 & 8,4 & 12 & 9,6 & 1,2 \\
\hline
\end{tabular}

Tableau 5 : résultats bactériologiques du lixiviat et des eaux des puits situés autour de la décharge publique (période sèche) et normes marocaines.

\begin{tabular}{ccccccccc}
\hline Paramètres & Lixiviat (période sèche) & P1 & P2 & P3 & P4 & P5 & P6 & normes \\
\hline Coli.T UFC/100 ml & 31600 & 3125 & - & 2050 & 16734 & 16467 & 33400 & 50 \\
Coli.F UFC/100 ml & 15200 & - & - & 500 & 5150 & 13966 & 600 & \\
Strep.F UFC/100 ml & 875 & 6800 & - & 625 & 150 & 100 & 800 & 20 \\
\hline
\end{tabular}

Tableau 6: matrice de corrélations des différents paramètres mesurés au niveau du lixiviat et des eaux des puits de la nappe R'Mel (Larache).

\begin{tabular}{|c|c|c|c|c|c|c|c|c|c|c|}
\hline & Od & CD & DCO & $\mathrm{NO}_{3}{ }^{-}$ & $\mathrm{Cl}$ & $\mathrm{SO}_{4}{ }^{2-}$ & $\mathrm{Ca}^{+}$ & $\mathbf{K}^{+}$ & $\mathbf{N a}^{+}$ & $\mathrm{Mg}^{2+}$ \\
\hline Od & 1,000 & & & & & & & & & \\
\hline CD &,- 462 & 1,000 & & & & & & & & \\
\hline DCO &,- 975 & ,600 & 1,000 & & & & & & & \\
\hline $\mathrm{NO}_{3}{ }^{-}$ &,- 949 &,- 105 & ,949 & 1,000 & & & & & & \\
\hline Cl & ,389 & ,949 &,- 105 & $-1,000$ & 1,000 & & & & & \\
\hline
\end{tabular}


H. ER-RAIOUI et al. / Int. J. Biol. Chem. Sci. 5(3): 1118-1134, 2011

\begin{tabular}{ccccccccccc}
\hline $\mathbf{S O}_{\mathbf{4}}{ }^{2-}$ &,- 975 &, 600 & 1,000 &, 949 &,- 105 & 1,000 & & & & \\
$\mathbf{C a}^{+}$ &,- 462 & 1,000 &, 600 &,- 105 &, 949 &, 600 & 1,000 & & \\
$\mathbf{K}^{+}$ &,- 462 & 1,000 &, 600 &,- 105 &, 949 &, 600 & 1,000 & 1,000 & \\
$\mathbf{N a}^{+}$ &,- 616 &, 900 &, 700 &, 105 &, 738 &, 700 &, 900 &, 900 & 1,000 \\
$\mathbf{M g}^{2+}$ &,- 462 & 1,000 &, 600 &,- 105 &, 949 &, 600 & 1,000 & 1,000 &, 900 & 1,000 \\
\hline
\end{tabular}

Tableau 7 : Comparaison des concentrations de métaux (exprimées en mg/l) dans les lixiviats de décharge de Larache par rapport aux autres nationaux et internationaux décharges.

\begin{tabular}{lcccccc}
\hline Site & $\begin{array}{c}\text { Larache } \\
\text { (Maroc) }\end{array}$ & $\begin{array}{c}\text { Rabat (Maroc) } \\
\text { Amhoud, (1997) }\end{array}$ & $\begin{array}{c}\text { El Jadida (Maroc) } \\
\text { Chofqi et al., 2004 }\end{array}$ & $\begin{array}{c}\text { Alger (Algérie) } \\
\text { Kerbachi and } \\
\text { Belkacemi (1994) }\end{array}$ & $\begin{array}{c}\text { Tiaret (Algérie) } \\
\text { Mekaikia et al., } \\
\text { 2007 }\end{array}$ & $\begin{array}{c}\text { Etueffont (France) } \\
\text { Khattabi (2002) }\end{array}$ \\
\hline Cu & 0.15 & 0,12 & 0,16 & 0,45 & - & 0,27 \\
Zn & 0.34 & - & 0,75 & 0,70 & 6,1 & 0,74 \\
Cd & 0.03 & 0,01 & 0,03 & - & - & 0,01 \\
Cr & 0.03 & 0,52 & 0,16 & 0,50 & 3 & 0,27 \\
Co & 0.04 & - & 0,21 & - & - & 0,06 \\
Fe & 16.65 & 23,00 & 24,00 & 12,30 & - & 2,63 \\
\hline
\end{tabular}




\section{DISCUSSION}

Les résultats des différentes analyses effectuées permettent de mettre l'accent sur la détérioration de la qualité des eaux de la nappe R'Mel. La provenance des contaminants du lixiviat est fort probable. Il est à signaler que jusqu'à 2005, les eaux de la nappe affichaient une bonne qualité minéralogique et bactériologique sur l'ensemble des stations échantillonnées, à l'exception des nitrates qui, par endroit, dépassent les normes de qualité admises (50 $\mathrm{mg} / \mathrm{l})$ (ABHL, 2005). Les résultats dégagés permettent de noter une variation des concentrations dans le temps et dans l'espace. En effet, pour la plupart des paramètres analysés, les concentrations pendant la période sèche sont plus élevées que pour la période humide, ce qui peut être expliqué par la diminution du niveau de la nappe pendant la période sèche et l'apport des eaux pluviales qui entraînent une dilution se traduisant par la diminution des taux en différents éléments. De même, les résultats mettent en relief une variation dans l'espace. Ainsi, la comparaison des concentrations relevées montrent que les puits P4, P5 et P6, situés dans le sens de l'écoulement du lixiviat et plus proches de la décharge, présentent, le plus souvent, des concentrations plus élevées en éléments analysés relativement aux autres puits situés plus loin de la décharge. Les mesures du $\mathrm{pH}$ indiquent des eaux de lixiviation acides $(\mathrm{pH}=$ 4.78) pendant la période sèche, ce qui peut avoir un impact négatif sur le sol et les eaux souterraines situées au dessous de la décharge. Les analyses réalisées montrent que le lixiviat représente une source de pollution à potentiel anoxique et réducteur vu la quasi absence d'oxygène dissous et les taux élevés en matières oxydables. La matrice de corrélation montre que les valeurs en oxygène dissous et en DCO sont inversement proportionnelles et donc négativement corrélées. Le coefficient de corrélation est de $-0,95$ (Tableau 6). La charge polluante est loin d'être négligeable et se traduit par des quantités importantes en matières oxydables avec des valeurs respectives de $44750 \mathrm{mg} / \mathrm{l}$ et $82000 \mathrm{mg} / \mathrm{l}$ pour la période humide et sèche. Cette charge suscite une demande importante en oxygène pour les réactions d'oxydation dépassant l'offre du milieu qui est quasiment nul et se traduit par les valeurs d'oxygène dissous souvent inférieures à $0.2 \mathrm{mg} / \mathrm{l}$. Ceci provoquera une succession de réactions de dégradation de la matière organique (Berner, 1984). En premier lieu, la dégradation se fait par les bactéries anaérobies utilisant les nitrates comme source d' $\mathrm{O}_{2}$ selon la réaction : $5 \mathrm{CH}_{2} \mathrm{O}+4 \mathrm{NO}_{3}{ }^{-} \rightarrow \mathrm{CO}_{2}+3 \mathrm{H}_{2} \mathrm{O}+2 \mathrm{~N}_{2}+$ $4 \mathrm{HCO}_{3}{ }^{-}$

Puis par les bactéries anaérobies sulfatoréductrices utilisant les sulfates comme source d' $\mathrm{O}_{2}$ selon la réaction :

$\mathrm{C}_{2} \mathrm{H}_{4} \mathrm{O}+\mathrm{SO}_{4} \longrightarrow 2 \mathrm{CO}_{2}+\mathrm{H}_{2} \mathrm{~S}+\mathrm{H}_{2} \mathrm{O}$

En ultime étape, les bactéries méthanogènes produisent le méthane, responsable des odeurs dégagées, à partir de la réduction de $\mathrm{CO}_{2}$ par $l^{\prime} \mathrm{H}_{2}$ ou en attaquant les acétates ou méthanol selon les réactions suivantes :

$\mathrm{CH}_{3} \mathrm{COOH} \longrightarrow \mathrm{CH}_{4}+\mathrm{CO}_{2}$

Il est à noter que la disponibilité et la réactivité de la matière organique déterminent l'ampleur des environnements redox au cours de la diagénèse (Banwart, 1999 ; Hartog et al., 2005). Les flux des accepteurs d'électron et les quantités d'espèces redox dans le sédiment sont aussi des facteurs déterminants (Von Gunten et Furrer, 2000 ; Andersen et al., 2001). En somme, cet état d'anoxie et de réduction permet la conservation de la charge polluante que reçoit la décharge publique, qui finira par s'infiltrer vers les poches souterraines à travers la percolation et constitue donc, une source de contamination située à quelques mètres au dessus.

Les conductivités élevées reflètent les grandes concentrations en calcium, magnésium, chlorures, potassium, sodium, sulfates et en azote kjeldahl (Tableaux 1, 2, 3 et 4). L'analyse de la matrice de corrélation permet de noter que les valeurs de la conductivité électrique (CE) sont parfaitement corrélées aux sulfates, aux chlorures, au sodium, au calcium au magnésium et au potassium. Les coefficients de corrélation sont respectivement de 0,80 , 
$0,96,0,9,1,1$ et 1 . La minéralisation est donc liée essentiellement aux sulfates et chlorures ainsi qu'aux autres éléments (Tableau 6). Le lixiviat est excessivement concentré en $\mathrm{Ca}^{2+}$, $\mathrm{K}^{+}, \mathrm{Na}^{+}$et $\mathrm{Mg}^{2+}$. Les concentrations moyennes, exprimées en $\mathrm{mg} / \mathrm{l}$, sont de 3225 , 8489,11114 et 240 . Les éléments fer, $\mathrm{Pb}$ et Cd montrent des concentrations respectives de $16650 \mu \mathrm{g} / \mathrm{l}, 280 \mu \mathrm{g} / \mathrm{l}$ et $30 \mu \mathrm{g} / \mathrm{l}$. Ces taux sont dans la gamme des concentrations rencontrées dans les lixiviats des décharges des pays industrialisés. En effet, en Angleterre, Danemark, Etats Unis, Grèce, Allemagne, Hong-Kong et Norvège, les concentrations en $\mathrm{Pb}$ et en $\mathrm{Cd}$ dans les lixiviats varient respectivement de 1 à $1400 \mu \mathrm{g} / \mathrm{l}$ et de 0,02 à $1950 \mu \mathrm{g} / \mathrm{l}$ (Mimides et Perraki, 2000 ; Baun et Christensen, 2004). Ceci reflète la composition des déchets ménagers et les contenus élevés en certains métaux. De manière générale, dans les aires urbaines, la production des déchets et leur mode d'élimination sont des activités qui produisent aussi de grandes quantités de polluants métalliques. La plupart des constituants des déchets urbains contiennent des métaux lourds tels que les piles ( $\mathrm{Hg}, \mathrm{Zn}, \mathrm{Pb}, \mathrm{Cd})$, les peintures $(\mathrm{Cr}, \mathrm{Cd}, \mathrm{Pb})$, les plastiques $(\mathrm{Cd}, \mathrm{Ni})$, les papiers cartons $(\mathrm{Pb})$ etc. (De Miquel, 2001). Des estimations des quantités de métaux enfouis dans des sites de stockage des déchets ménagers en Norvège montrent des quantités importantes en $\mathrm{Pb}$ (133 tonnes), en $\mathrm{Cu}$ (1,60 tonnes), en $\mathrm{Cr}$ (6,25 tonnes) et en $\mathrm{Fe}$ (2617,5 tonnes) par rapport à une masse totale des déchets enfouis équivalente à 2955000 tonnes (Oygard et al., 2004). Exprimés en $\mu \mathrm{g} / \mathrm{l}$, les concentrations en $\mathrm{Pb}, \mathrm{Cu}, \mathrm{Cr}$ et $\mathrm{Fe}$ sont respectivement de $45,0,54,2,12$ et 886 . A titre d'exemple, en Algérie, les lixiviats de la décharge de la ville de Tiaret, située à $150 \mathrm{~km}$ au Sud-Ouest de Mostaganem (Algérie occidentale), entraînent une dégradation de la qualité des eaux souterraines. Les analyses effectuées sur des échantillons de lixiviat montrent des concentrations importantes en chrome $(3000 \mu \mathrm{g} / \mathrm{l})$, zinc $(6100 \mu \mathrm{g} / \mathrm{l})$, plomb $(900 \mu \mathrm{g} / \mathrm{l})$, cadmium $(500 \mu \mathrm{g} / \mathrm{l})$. Les analyses montrent aussi un DCO de $1230 \mathrm{mg} / \mathrm{l}$ et une contamination microbiologique (Mekaikia mokhtaria et al., 2007).

L'analyse du lixiviat montre aussi une forte contamination bactériologique avec des valeurs de $316000 \mathrm{UFC} / 100 \mathrm{ml}$ pour les coliformes totaux, $152000 \mathrm{UFC} / 100 \mathrm{ml}$ pour les coliformes fécaux et $875 \mathrm{UFC} / 100 \mathrm{ml}$ pour les streptocoques fécaux.

Au niveau des puits, les analyses des eaux évoquent une mauvaise qualité avec des concentrations qui dépassent, pour certains éléments, les valeurs maximales admises pour une eau distribuée (MEM, 2002). Pour la DCO, à l'exception des eaux des puits P3 et P6 qui montrent des DCO légèrement supérieures aux normes, les eaux ne sont pas trop chargées en matières oxydables. Les quantités ne dépassent pas $20 \mathrm{mg} / \mathrm{l}$ d'où l'oxygénation normale des eaux en question. Pour le bilan ionique, les concentrations en calcium, chlorures et nitrates atteignent respectivement des valeurs de $252 \mathrm{mg} / 1,248.5$ $\mathrm{mg} / \mathrm{l}$ et $114 \mathrm{mg} / \mathrm{l}$. Les teneurs élevées en calcium varient entre $116 \mathrm{mg} / \mathrm{l}$ et $184 \mathrm{mg} / \mathrm{l}$ pour la période humide et entre $170 \mathrm{mg} / \mathrm{l}$ et $252 \mathrm{mg} / \mathrm{l}$ pour la période sèche. Ces valeurs dépassent souvent les normes admises pour une eau de consommation. Ceci témoigne d'une pollution des eaux de la nappe par le calcium provenant probablement de la décharge à travers le lixiviat et qui englobe une grande quantité d'os de poisson provenant de l'activité industrielle de conserve de poisson. Les analyses des chlorures montrent, par endroit, des concentrations dépassant la norme admise qui est de $200 \mathrm{mg} / \mathrm{l}$. C'est le cas des eaux des puits P1, P2 et P6. Les chlorures pourraient provenir de la percolation des rejets industriels à travers les terrains salés (Bremond et Perredon, 1979 ; Kholtei et al., 2003). Ils peuvent aussi provenir de l'écoulement des eaux d'irrigation (Bond et Straud, 1973 ; Lhadi et al., 1996).

Les nitrates montrent des taux élevés pouvant atteindre $114 \mathrm{mg} / \mathrm{l}$ pour la période sèche. Les concentrations dépassent les normes au niveau de tous les puits pendant toute l'année (Tableaux 1 et 2). Cette contamination des eaux souterraines peut provenir de la charge polluante de la décharge, qui ne dispose que de $12 \mathrm{mg} / \mathrm{l}$ de nitrates en moyenne, par une réaction biochimique de genèse des nitrates à partir des composés organiques azotés selon la réaction suivante : 
Composé organique azoté $\longrightarrow$ Ammonium $\mathrm{NH}_{4} \longrightarrow$ Nitrites $\mathrm{NO}_{2}{ }^{-} \longrightarrow$ Nitrates $\mathrm{NO}_{3}{ }^{-}$

Il est à noter que les composés azotés sont présents dans la décharge en grande quantité comme en témoigne les concentrations en azote kjeldahl correspondantes à 8000 mg/l. Parallèlement, la pollution par les nitrates peut être aussi due et en partie à l'utilisation excessive des fertilisants azotés, à l'élevage et au mode d'irrigation. Les mêmes résultats ont été confirmés par des études réalisées dans d'autres régions du Maroc (Lhadi et al., 1996 ; Amhoud, 1997 ; Laftouhi et al., 2003) et en Europe (Exner et al., 1985 ; Baun et al., 2000). Dans ces domaines, les principales sources de contamination par les nitrates sont des engrais et des eaux usées domestiques. Des analyses effectuées sur des échantillons d'eau de 131 puits à travers la zone des Niayes au Sénégal montrent une teneur en nitrates supérieure à $50 \mathrm{mg} / \mathrm{l}$ (Sall et Vanclooster, 2009).

Les analyses des métaux montrent des eaux chargées en $\mathrm{Pb}$, en $\mathrm{Cd}$ et, par endroit, en $\mathrm{Cr}$ et en $\mathrm{Fe}$, ce qui reflète le phénomène de contamination des eaux souterraines qui se produit à partir du lixiviat chargé en contaminants. Ceci peut aussi être expliqué par des pratiques agricoles dans la région qui utilisent des fongicides, des herbicides et des insecticides pour la lutte chimique (El Bakouri, 2006). Les concentrations peuvent atteindre $280 \mu \mathrm{g} / \mathrm{l}$ pour le $\mathrm{Pb}, 30 \mu \mathrm{g} / \mathrm{l}$ pour le $\mathrm{Cd}, 30$ $\mu \mathrm{g} / \mathrm{l}$ pour le $\mathrm{Cr}$ et $16650 \mu \mathrm{g} / \mathrm{l}$ pour le $\mathrm{Fe}$, ce qui dépassent les normes admises, mais restent dans la gamme des concentrations révélées dans plusieurs régions du Maroc et ailleurs (Tableau 7). En effet, les lixiviats des décharges des villes de Rabat, El Jadida, Alger, Tiaret et Etueffont montrent des teneurs en $\mathrm{Cd}, \mathrm{Cr}$ et $\mathrm{Fe}$ qui varient de $10 \mu \mathrm{g} / \mathrm{l}$ à $30 \mu \mathrm{g} / \mathrm{l}$, de $30 \mu \mathrm{g} / \mathrm{l}$ à $300 \mu \mathrm{g} / \mathrm{l}$ et de 2630 $\mu \mathrm{g} / \mathrm{l}$ à $24000 \mu \mathrm{g} / \mathrm{l}$ respectivement. Les métaux, dont les teneurs ne dépassent pas les limites admises, peuvent constituer un danger pour la santé. En effet, ces éléments par bioaccumulation et bioamplification, peuvent se trouver à des doses toxiques à un certain niveau de la chaine trophique, surtout que les eaux des puits sont utilisées pour l'abreuvage des animaux et l'irrigation des cultures dans la région. En effet, les métaux lourds, plus particulièrement le Plomb, le Zinc et le Cuivre, font partie des cas de pollution représentant un problème environnemental majeur. Ils peuvent migrer dans les eaux souterraines, s'accumuler dans la chaîne alimentaire et présenter des risques pour la santé humaine (Jourdan et al., 2005). Mais La migration des métaux lourds dans les sols et l'infiltration vers les eaux, restent tributaire de plusieurs processus tels que la solubilisation ou la précipitation qui sont fonction $\mathrm{du} \mathrm{pH}$, la formation de complexes organométalliques, l'adsorption des ions ou des molécules à la surface des grains de matrice poreuse, phénomène d'échange d'ions etc. (Lagier, 2000). Par conséquent, la majorité des polluants métalliques susceptibles d'atteindre les eaux souterraines peuvent subir, lorsqu'ils transitent par le sol, des transformations biophysico-chimiques, qui auront pour effet soit de les immobiliser ou de les retarder, ou de favoriser leur solubilisation et leur transport par les eaux d'infiltration (Février, 2001). Il est aussi à noter que l'atténuation naturelle qui correspond à l'ensemble des processus de dispersion, dilution, volatilisation, adsorption, solubilisation, mécanismes de stabilisation ou de destruction des polluants pouvant se produire dans les sols ou dans les eaux souterraines et visant à réduire la toxicité, la mobilité, le volume ou les concentrations des contaminants dans ces milieux (USEPA, 1999; Mulligan et Yong, 2004), peut expliquer les concentrations faibles relevées au niveau des eaux des puits. En effet, sur la base d'analyses physico-chimiques, Yanful et al. (1988) étudiant la répartition des phases porteuses d'éléments métalliques à l'aplomb immédiat des déchets, montrent que la teneur en $\mathrm{Pb}$, qui est de $75 \mu \mathrm{g} / \mathrm{l}$ à l'interface déchets/couche argileuse, diminue rapidement dès les premiers centimètres de profondeur. A $10 \mathrm{~cm}$, la teneur en $\mathrm{Pb}$ n'est que de quelques $\mu \mathrm{g} / \mathrm{l}$. Les auteurs notent aussi, un comportement similaire pour le $\mathrm{Zn}$ et le $\mathrm{Cu}$. L'influence des fractions argileuses et 
carbonatées du sol ainsi que sa teneur en matière organique sur la rétention des métaux a été mis en évidence par Howari en 2004 suite à une étude géochimique portant sur la répartition des éléments métalliques $(\mathrm{Cd}, \mathrm{Ni}$, $\mathrm{Pb}, \mathrm{Zn}, \mathrm{Cu}$ et $\mathrm{Cr}$ ) dans les sols aux alentours de la décharge d'Al-Ain aux Emirats Arabes Unis.

Les analyses bactériologiques qui ont porté sur les coliformes totaux, les coliformes fécaux et les streptocoques fécaux dans les eaux souterraines montrent des valeurs respectives de $2050 \mathrm{UFC} / 100 \mathrm{ml}$ à 33400 $\mathrm{UFC} / 100 \mathrm{ml}$, de $500 \mathrm{UFC} / 100 \mathrm{ml}$ à 13966 $\mathrm{UFC} / 100 \mathrm{ml}$ et de $100 \mathrm{UFC} / 100 \mathrm{ml}$ à 68000 $\mathrm{UFC} / 100 \mathrm{ml}$, dépassant ainsi et de loin les normes admises, d'où une contamination bactériologique bien marquée. Des niveaux de contamination pareils ont été signalés au niveau des eaux de dix puits de la commune des Mzamza au nord de la ville de Settat. Les résultats obtenus confirment une contamination microbiologique. On y trouve jusqu'à 6778 de coliformes fécaux/100ml et jusqu'à 9952 streptocoques fécaux $/ 100 \mathrm{ml}$ (Hassoune et al., 2010). A Marrakech, au centre du Maroc, la nappe phréatique héberge de fortes densités en flore totale et en indicateurs de la contamination fécale (Boutin et Dias, 1887). Dans la région de Jbilet au Sud de Marrakech (Maroc), l'activité anthropique intense et la perméabilité des terrains géologiques rendent la nappe phréatique vulnérable. Les puits situés dans la zone de la décharge publique de la ville de Marrakech et au sein d'une agglomération périurbaine montrent une eau de qualité détériorée. Les résultats des analyses bactériologiques de l'eau montrent une contamination fécale importante (Hallam et al., 2008). En effet, la contamination du sol et des eaux souterraines en raison des teneurs élevées en matière organique et en microorganismes d'origine fécale peut atteindre $6 \times 10^{7} \mathrm{CFU} / 100 \mathrm{ml}$ (Njine et al., 2001). L'origine des bactéries dans les eaux souterraines est souvent discutée, mais selon plusieurs auteurs, la majeure partie des bactéries souterraines sont dérivées des bactéries infiltrées par percolation (Mayer et al., 1997 ; Dzeda et al., 1998). Une nappe phréatique est d'autant plus vulnérable que son sommet est proche de la surface et que les terrains surmontant l'aquifère sont perméables (Boutin, 1987). En outre, les rejets d'élevage et le fumier utilisé comme fertilisant contribuent à la pollution des eaux souterraines (Bridgman et al., 1995).

Dans la région et non loin de Larche, la qualité des eaux souterraines des bassins du Loukkos, du Tangérois et des Côtiers méditerranéens, situés au Nord du Maroc, reste fonction des actions anthropiques. La qualité est bonne à moyenne au niveau des nappes Amsa et Azla, mauvaise au niveau des nappes Charf El Akkab, Laou et Oulad Ogbane et très mauvaise au niveau des nappes Martil, Neckor, Smir et Negro (ABHL, 2005) (Fig. 1). Dans d'autres régions du Maroc, la situation n'est pas meilleure. La nappe M'nasra, située au nord de la ville de Kénitra, présente une forte contamination bactériologique et par les nitrates, reflétant ainsi, l'impact des rejets domestiques et urbains (Bricha et al., 2007). Dans la communauté des Mzamza, située au niveau de la ville de Settat (Sud de Casablanca), caractérisée par une potentialité agricole importante, les eaux de la nappe sont sujettes à plusieurs possibilités de contamination. Les concentrations des éléments correspondant aux principaux polluants et indicateurs de pollution sont élevées dans les eaux usées et dans les eaux souterraines (El Asslouj et al., 2007). Plusieurs facteurs déterminants ont été mis en évidence dans la contamination des eaux souterraines par ces polluants, à savoir : l'apport en fertilisants azotés, la nature du sol, la lithologie, la perméabilité de l'aquifère et la distance du puits par rapport aux sources de pollution (El Asslouj et al., 2007). A El Jadida, la situation n'est pas différente. La décharge, l'une des nombreux sites incontrôlés et sans revêtement de fond, constitue un impact négatif sur les eaux souterraines (Chofqi et al., 2004). Les analyses géochimiques montrent une importante charge polluante véhiculée par le lixiviat de décharge vers les eaux du fond. Au niveau des eaux des puits à proximité de la 
décharge, les analyses montrent une forte teneur en chlorures $(1620 \mathrm{mg} / \mathrm{l})$, en sulfates (1000 mg/l) et en éléments métalliques (15-25 $\mu \mathrm{g} / \mathrm{l}$ en cadmium et $60-100 \mu \mathrm{g} / \mathrm{l}$ en chrome) (Chofqi et al., 2004). D'autres études dans la région de Rabat (Amhoud, 1997) et dans la région de Belfort (Khattabi, 2002), montrent que l'impact des lixiviats de décharge sur la qualité des eaux souterraines est important, surtout pour les puits situés à proximité de la décharge. A Tiaret en Algérie, les eaux des puits montrent des concentrations élevées en certains métaux pouvant atteindre $2930 \mu \mathrm{g} / \mathrm{l}$ pour le $\mathrm{Zn}, 750 \mu \mathrm{g} / \mathrm{l}$ pour le $\mathrm{Cr}, 150 \mu \mathrm{g} / \mathrm{l}$ pour le $\mathrm{Cu}$ et $340 \mu \mathrm{g} / \mathrm{l}$ pour le $\mathrm{Pb}$, reflétant ainsi, l'influence des lixiviats qui s'écoulent à partir de la décharge de la ville.

A travers le monde et dans beaucoup de pays, des nappes d'eaux phréatiques sont déjà fortement polluées par des substances chimiques et la situation est encore plus alarmante dans les pays en voie de développement où les eaux souterraines montrent la présence de métaux lourds, bactéries, $\mathrm{NO}_{3}, \mathrm{Cl}^{-}, \mathrm{NO}_{3}, \mathrm{NH}_{4}$, et coliformes fécaux. C'est le cas des eaux souterraines des régions et villes de Madras en Inde (Howard et Beck, 1993), de Taejon en Corée (Jeong, 2001), de Sana'a au Yémen (Foppen, 2002), de Port-au-Prince à Haïti (Emmanuel, 2004) et du Harare au Zimbabwe (Zingoni et al., 2005).

Face à cette pollution engendrée par la décharge sur la nappe phréatique et par conséquence sur les populations et l'environnement dans la région, il est nécessaire d'envisager l'aménagement du site ou le transfert de la décharge à un site contrôlé et d'étanchéité efficace empêchant toute diffusion $\mathrm{du}$ lixiviat vers les eaux superficielles et souterraines. L'installation d'une plate forme de traitement visant la valorisation des déchets par compostage et l'élimination du lixiviat par compaction s'avère nécessaire. Cela suppose la mise en marche d'une station pilote de compostage pour la production du composte utile pour la fertilisation des sols accompagnée d'un compacteur permettant l'élimination du lixiviat vers un bassin de collecte. D'autres processus de traitement peuvent être envisagés. Ils reposent essentiellement sur des phénomènes d'évaporation et d'adsorption. Le traitement par évaporation s'avère efficace pour l'élimination du lixiviat utilisant la chaux comme source d'énergie. L'adsorption, qui se base sur une réaction de fixation des ions libres à la surface de la substance, est d'une grande utilité pour le rabattement de la charge polluante. Des études réalisées sur le lixiviat, utilisant la diatomite ou le charbon actif comme adsorbant ont montré une diminution de la DCO de 90 à $95 \%$.

\section{Conclusion}

La décharge publique de Larache constitue un modèle type de détérioration des ressources en eaux par le lixiviat. La caractérisation physico-biogéochimique du lixiviat et l'évaluation de l'impact sur la nappe phréatique située au dessous permettent de qualifier le lixiviat comme une source de contamination à potentiel anoxique et réducteur. La décharge de Larache est de type non contrôlée en raison de la perméabilité du site et l'absence de réseau de drainage et du dispositif du récupérateur et d'épuration du lixiviat. Les analyses du lixiviat montrent des eaux de type anoxique et réductrice, chargées chimiquement et bactériologiquement et dont les concentrations en ions, fer, $\mathrm{O}_{2}$ dissous, DCO, coliformes totaux, coliformes fécaux et streptocoques fécaux dépassent les normes des rejets directs dans le milieu naturel. Le lixiviat représente alors une source de contamination potentielle pour son environnement et surtout pour la nappe phréatique se trouvant au dessous de la décharge. L'évaluation de la qualité de la nappe, à travers les analyses des eaux des puits, montre la présence de certains éléments polluants présents dans les lixiviats analysés. On note une contamination bactériologique et des fortes concentrations en certains éléments : calcium, chlorures, nitrates et en certains métaux. Le vecteur de transfert de cette pollution est l'infiltration à cause de la nature du sol sur lequel repose la décharge, correspondant à un sable dunaire caractérisé 
par une grande perméabilité. Ceci est encore favorisé par une pluviométrie importante avoisinant $700 \mathrm{~mm} / \mathrm{an}$. Cette pollution de la nappe aura un effet négatif sur les populations de la région R'Mel faisant partie de la Province de Larache qui utilisent les eaux de puits pour la consommation, l'irrigation et l'abreuvage de leurs animaux.

\section{REFERENCES}

ABHL (Agence du Bassin Hydraulique du Loukkos). 2003. Nappe de R'Mel: situation des ressources en eau, état de mobilisation et perspectives de développement, Rapport interne.

ABHL (Agence du Bassin Hydraulique du Loukkos). 2005. Etat de la qualité des ressources en eau dans la région hydraulique du Loukkos (année 20032004). Rapport interne.

Amhoud S. 1997. Apports de la géologie et de l'hydrogéologie à l'étude de l'impact de la décharge d'Oued Akrech sur les ressources en eau. Thèse de Doctorat, Université de Rabat, Rabat, p. 204.

Andersen MS, Larsen F, Postma D. 2001. Pyrite oxidation in unsaturated aquifer sediments. Reaction stoichiometry and rate of oxidation. Env. Sci. Technol., 35: $4074-4079$.

Banwart SA. 1999. Reduction of iron (III) minerals by natural organic matter in groundwater. Geochimica et Cosmochimica Acta, 63(19/20): 2919 2928.

Baun A, Jensen SD, Bjerg L, Christensen TH, Nyholm N. 2000. Toxicity of organic chemical pollution in groundwater down gradient of a landfill (Grindsted, Denmark). Environmental and Technology, 34: 647-1652.

Baun DL, Christensen TH. 2004. Speciation of heavy metals in landfill leachates : a review. Waste Management and Research, 22: 3-23

Berner R. 1984. Sedimentary pyrite formation : an update. Geochimica et Cosmochimica Acta, 48(4): 605-615.
Bond RG , Straud CP. 1973. Handbook of Environnemental Control (end). CRC Press: Cleveland.

Boutin C. 1987. L'eau des nappes phréatiques superficielles, une richesse naturelle vitale mais vulnérable: L'exemple des Zones rurales du Maroc. Sci. Eau, 6(3): $357-65$.

Boutin C, Dias N. 1987. Impact de l'épandage des eaux usées de la ville de Marrakech sur la nappe phréatique. Bull. Fac. Sci. Marrakech (Sci. Vie), 3: 525.

Bremond R, Perredon C. 1979. Paramètres de la Qualité des Eaux (edn). Ministère de l'environnement Press : Paris.

Bricha S, Ounine K, Oulkheir S, El haloui N, Attarassi B. 2007. Etude de la qualité physicochimique et bactériologique de la nappe phréatique M'nasra (Maroc). Afrique Science, 3(3): 391-404.

Bridgman SA, Robertson RMP, Syed Q, Speed N, Andrews N, Hunter PR. 1995. Outbreak of Cryptosporidiosis Associated With a Disinfected Groundwater Supply. Epidemiology and Infection, 115(3): 555566.

Chofqi A, Younsi A, Lhadi E, Jacky Mania J, Mudry J, Veron A. 2004. Environmental impact of an urban landfill on a coastal aquifer (El Jadida, Morocco). Journal of African Earth Sciences, 39: 509-516.

Christensen TH, Kjeidsen R, Bjerg PL, Jensen DL, Christensen J, Bauna A, Albrechtsen HJ, Heron G. 2001. Biogeochemistry of landfill leachate plumes. Application Geochemistry, 16: 659-718.

Danielpol D, Griebler C, Gunatilaka A, Notenboom J. 2003. Present state and future prospect for groundwater ecosystems. Environnemental Conservation, 30(2): 1-27.

DE Miquel G. 2001. Rapport sur les Métaux Lourds et leurs Effets sur l'Environnement et la Santé (edn). Sénat: Paris.

Dzeda B, Kaiser M, Mach S. 1998. Bacterial and groundwater. Soil and groundwater pollution. Civil Engineering Dept., 
Virginia Tech. http://www.cee.vt.edu/ programareas/environmental/teach/ gwprimer/bacteria.html (accessed 25th March 2005).

El Bakouri H. 2006. Développement de nouvelles techniques de détermination des pesticides et contribution à la réduction de leur impact sur les eaux par utilisation des substances organiques (S.O. N.). thèse de Doctorat, Univerité Abdelmalek Essâadi, Tanger, p. 148.

El Asslouj J, Kholtei S, El Amrani N, Hilali A. 2007. Analyse de la qualité physicochimique des eaux souterraines de la communauté des Mzamza, au voisinage des eaux usées. Afrique Science, 3(1): 109-122.

EmmanueL E. 2004. Evaluation des risques sanitaires et écotoxicologiques liés aux effluents hospitaliers. Thèse de Doctorat, Université de Lyon, Lyon, p. 260.

Exner ME, Spallding RF, Lindeau CW. 1985. Groundwater contamination and well Construction in Southeast Nebraska. Grounwater, 23: 26-34.

Février L. 2001. Transfert d'un mélange Zn$\mathrm{Cd}-\mathrm{Pb}$ dans un dépôt fluvio-glaciaire carbonaté. Approche en colonnes de Laboratoire. Thèse de Doctorat, Université de Lyon, Lyon, p. 312.

Foppen JWA. 2002. Impact of high-strength wastewater infiltration on groundwater quality and drinking water supply: the case of Sana'a, Yemen. Journal of Hydrology, 263(1-4): 198-216.

Hallam F, Yacoubi-Khebiza M, Oufdou K, Boulanouar M. 2008. Qualité des eaux souterraines dans une région aride du maroc: impact des pollutions sur la biodiversité et relations crustacésbactéries d'intérét sanitaire. Environmental Technology, 28(11): 1179-1189.

Harter T, Davi H, Mathews MC, Meyer RD. 2002. Shallow groundwater quality on dairy farms with irrigated forage crops. $J$. Cont. Hydrol., 55: 287-315.

Hassoune E, El kettani S, Koulaliet Y, Bouzidi A. 2010. Contamination bacteriologique des eaux souterraines par les eaux usees de la ville de settat, Maroc. Rev. Microbiol. Ind. San. et Environn., 4(1): 1-21.

Hudak PF. 2000. Regional trends in nitrate content of Texas groundwater. J. Hydrol., 228: 37-47.

Hartog N, Griffioen J, Van bergen PF. 2005. Depositional and paleohydrogeological controls on the distribution of organic sediments. Chemical Geology, 216: 113 131.

Howard KWF, Beck PJ. 1993. Hydrogeochemical implications of groundwater contamination by road deicing chemicals. Journal of Contaminant Hydrology, 12: 245-268.

Howari FM. 2004. Heavy metal speciation and mobility assessment of arid soils in the vicinity of Al Ain landfill, United Arab Emirates. Int. J. Environment and Pollution, 22(6): 721-731.

Jeong CH. 2001. Effect of land use and urbanization on hydrochemistry and contamination of groundwater from Teajon area, Korea. Journal of Hydrology, 253: 194-210.

Jourdan B, Piantone P, Lerouge C, Guyonnet D. 2005. Atténuation des métaux à l'aval de sites de stockage de déchets (Synthèse bibliographique). BRGM/RP 54417, 110 p.

Khattabi H, Lotfi A, Mania J. 2002. Evaluation de l'impact des lixiviats d'une décharge d'ordures ménagères sur la qualité physicochimique et bactériologique des eaux d'un ruisseau de Franche-Comté. Déchets-Sciences et Techniques, 24: 1-4.

Kholtei S, Bouzidi A, Bonin M, Fekhaoui M, Anane R, Sbai K, Creppy E. 2003. Contamination des eaux souterraines de la plaine de Berrechid dans la région de la Chaouia au Maroc par les métaux lourds dans les eaux usées : effets de la pluviométrie. Vecteur Environnement, 36(5): 68-80.

Kross BC, Ayebo AD, Fuortes LJ. 1992. Methemoglobinemia : nitrate toxicity in 
rural America. Am. Fam. Physician, 46: 183-188.

Laftouhi NE, Vanclooster M, Jalal M, Witam O, Aboufirassi M, Bachir M, Persons E. 2003. Groundwater nitrate pollution in the Essaouira Basin (Morocco). C. R. Geosci., 335: 307-317.

Lagier T. 2000. Etude des macro molécules de lixiviat : Caractérisation et comportement vis-à-vis des métaux. Thèse de Doctorat, Université de Poitiers, Poitiers, p. 189.

Lhadi EK, Mountadar M, Tounsi A. 1996. Pollution par les nitrates des eaux souterraines de la zone littorale de la province d'el Jadida (Maroc). Hydrogéologie, 3: 21-23.

Mayer AS, Carrière PPE, Gallo C, Pennel KD, Taylor TP, Williams GA, Zhong L. 1997. Groundwater quality. Wat. Environ. Res., 69(4): 778- 844.

Mekaikia MM, Belabbed B, Djabri 1, Hani A, Laour R. 2007. Caractéristiques de la décharge publique de la ville de Tiaret et son impact sur la qualité des eaux souterraines. Courrier du Savoir, 8: 9399.

MEM : Ministère de l'Aménagement du Territoire, de l'Eau et de l'Environnement. 2002. Normes marocaines, Bulletin officiel du Maroc, $N^{\circ} 5062$ du 30 ramadan 1423. Rabat.

Mimides T, Perraki T. 2000. Evaluation of the attenuating properties of selected Greek clays for toxic inorganic elements in landfill sites. The Science of the Total Environment, 253: 1-13.

Mulligan CN, Yong RN. 2004. Natural attenuation of contaminated soils. Environment International, 3(4): 587601.

Naifar EM. 1996. La situation actuelle de l'élimination des ordures ménagères de l'agglomération de Rabat. Etude de l'impact des rejets liquides de la de'charge d'Akrach sur l'Environnement.
Thèse de Doctorat, Université de Rabat, Rabat, 80 p.

Njine T, Monkiedje A, Nola M, Sikati-Foko V. 2001. Evaluation de la charge polluante et de la charge bactérienne des rejets des stations d'épuration à boues activées à Yaounde (Cameroun). Cahiers Santé, 11: 79-84.

Oygard JK, Amund MA, Gjengedal E. 2004. Estimation of the mass-balance of selected metals in four sanitary landfills in Western Norway, with emphasis on the heavy metal content of the deposited waste and the leachate. Water Research, 38(12): 2851-2858.

Rodier J. 1996. L'Analyse de l'Eau: Eaux Naturelles, Eaux Résiduaires, Eau de Mer : Physico-chimie, Bactériologie et Biologie (edn). The Dunod : Paris.

Sall M, Vanclooster M. 2009. Assessing the well water pollution problem by nitrates in the small scale farming systems of the Niayes region (Senegal). Agricultural Water Management, 96: 1360-1368.

USEPA. 1999. Use of Monitored Natural Attenuation at Superfund, RCDA Corrective Action, and Underground Storage Tank Sites. Final OSWER Directive 9200. P. 4-17

Von gunten U, Furrer G. 2000. Steady state modelling of biogeochemical processes in columns with aquifer material: 2 . Dynamics of iron interactions. Chemical Geology, 167: 271 - 284.

Yanful EK, Quigley RM, Nesbitt HW. 1988. Heavy metal migration at a landfill site, Sarnia, Ontario, Canada-2: metal partitioning and geotechnical implications. Applied Geochemistry, 3: 623-629.

Zingoni E, Love D, Magadza C, Moyce W, Musiwa K. 2005. Effects of a semiformal urban settlement on groundwater quality Epworth (Zimbabwe) : Case study and groundwater quality zoning. Physics and Chemistry of the Earth, 30: 680-688. 